PROCEEDINGS OF THE

AMERICAN MATHEMATICAL SOCIETY

Volume 125, Number 9, September 1997, Pages 2555-2558

S 0002-9939(97)03933-6

\title{
A CHARACTERIZATION OF RINGS IN WHICH EACH PARTIAL ORDER IS CONTAINED IN A TOTAL ORDER
}

\author{
STUART A. STEINBERG
}

(Communicated by Lance W. Small)

\begin{abstract}
Rings in which each partial order can be extended to a total order are called $O^{*}$ - rings by Fuchs. We characterize $O^{*}$ - rings as subrings of algebras over the rationals that arise by freely adjoining an identity or one-sided identity to a rational vector space $N$ or by taking the direct sum of $N$ with an $O^{*}$ field. Each real quadratic extension of the rationals is an $O^{*}$ - field.
\end{abstract}

A ring $R$ is called an $O^{*}$ - ring if each of its ring partial orders can be extended to a total order of $R$. Two of the problems in the list at the back of Fuch's book [4] concern $O^{*}$ - rings.

(A) Establish ring theoretical properties of $O^{*}$ - rings.

(B) Does there exist a polynomial identity which forces each totally ordered ring that satisfies it to be an $O^{*}$ - ring?

These problems were perhaps motivated by the well-known fact that each torsionfree abelian group is an $O^{*}$ - group. Recently, Kreinovich [7] has shown that (B) has a negative answer in the sense that if $f\left(x_{1}, \cdots, x_{n}\right)=0$ is such an identity, then each $O^{*}$ - ring that satisfies it must be trivial; that is, $R^{2}=0$. In the process of showing this he noted that an $O^{*}$ - ring has two very restrictive properties: it is algebraic over $\mathbb{Z}$ and each nilpotent element has index at most two. To see this first recall that the partial order in a partially ordered ring $R$ is determined by its positive cone $R^{+}=\{x \in R: x \geq 0\}$; we will refer to such a positive cone as a partial order of $R$. Now if $a$ is an element of an $O^{*}-\operatorname{ring} R$ that is not algebraic over $\mathbb{Z}$, then $\mathbb{Z}^{+}\left[-a^{2}\right]$ is a partial order of $R$ which is not contained in any total order of $R$. Also, if $a \in R$ is nilpotent of index $n>2$ let $b=-a^{n-2}$ if $n$ is even and let $b=-a^{n-1}$ if $n$ is odd. Then $\mathbb{Z}^{+} b$ is a partial order of $R$ that is not contained in any total order of $R$.

Clearly, each subring of an $O^{*}$ - ring $R$ is an $O^{*}$ - ring, and its divisible hull $d(R)$ is also an $O^{*}$ - ring. For if $P$ is a partial order of $d(R)$ and $T$ is a total order of $R$ which contains $P \cap R$, then $d(T)=\{x \in d(R): \exists n>0$ with $n x \in T\}$ is a total order of $d(R)$ which contains $P$. Consequently, in this paper we will deal exclusively with algebras over the rationals $\mathbb{Q}$. All such $O^{*}$ - algebras are determined in the

Theorem. If $R$ is an $O^{*}$ - algebra, then there is a $\mathbb{Q}$ - vector space $N$ such that $R$ is (isomorphic to) one of the following algebras.

Received by the editors April 9, 1996.

1991 Mathematics Subject Classification. Primary 06F25.

(C)1997 American Mathematical Society 
(i) $R=F \oplus N$ (algebra direct sum) where $F$ is a subfield of the reals that is algebraic over $\mathbb{Q}$ and $N^{2}=0$.

(ii) $R=\left(\begin{array}{cc}\mathbb{Q} & N \\ 0 & 0\end{array}\right)$ or the dual $\left(\begin{array}{cc}\mathbb{Q} & 0 \\ N & 0\end{array}\right)$.

(iii) $R=\left\{\left(\begin{array}{ll}a & b \\ 0 & a\end{array}\right): a \in \mathbb{Q}, b \in N\right\}$.

Moreover, each of these algebras is an $O^{*}$ - algebra where in (i) $F$ is an $O^{*}$-field.

Proof. If $G$ and $H$ are po-groups with positive cones $P_{G}$ and $P_{H}$ respectively, then

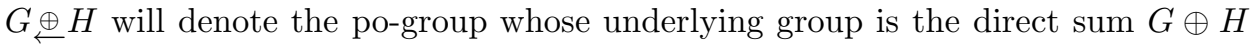
and whose positive cone is $\left\{g+h: 0 \neq h \in P_{H}\right.$, or $h=0$ and $\left.g \in P_{G}\right\}$; and $G \stackrel{\oplus \rightarrow}{\rightarrow}$ will denote the same group ordered similarly but with $G$ dominating. The same arrow notation will be used for other lexicographic orderings even if there are more than two summands.

In a totally ordered ring the set $N$ of nilpotent elements is an ideal and the quotient modulo $N$ is a totally ordered domain [4, p.130]. Assume that $R^{2} \neq 0$. Then $R$ has a nonzero idempotent $e$, and by Albert's theorem [1] $R / N$ is a field. Since $R / N$ can be embedded in the real closure of $\mathbb{Q}[6$, p.285] we may assume that it is a subfield of the reals.

Suppose first that $R$ is unital and $N \neq 0$. If $F=R / N$ is a proper extension of $\mathbb{Q}$ let $\left\{a_{i}: i \geq 1\right\}$ be a basis of $\mathbb{Q} F$ with $a_{1}=1$. Then $F=\stackrel{\oplus}{\rightarrow} i \geq 1 \mathbb{Q} a_{i}$ is a totally ordered group. Since $N^{2}=0 \quad N$ is a vector space over $F$. Let $0 \neq x \in N$. Then $F^{+} x$ is a partial order of $R$ and hence is contained in a total order $T$ of $R$. This total order induces a total order $T_{F}$ of the field $F$. Since $\left(F, T_{F}\right)$ is archimedean, $T_{F} \nsubseteq F^{+}$. Let $a \in T$ with $a+N \notin F^{+}$. Then $(a+N) x=a x \in T \cap F x=F^{+} x$ yields the contradiction that $a+N \in F^{+}$. Thus $F=\mathbb{Q}$ and $R=\mathbb{Q} 1+N$ is isomorphic to a ring of type (iii).

Suppose now that $R$ is not unital. Since the left and right annihilator ideals of $R$ are convex ideals one of them is contained in the other. Suppose that the right annihilator $r(R)$ is contained in the left annihilator $l(R)$. According to [2, Theorem 9.4.15] (also see $[5,2.4]$ ) the Pierce decomposition of $R$ is $R=B \oplus C \oplus D$ where $B=e R e, D=r(R)=(1-e) R, C=e R(1-e)$ and $C \oplus D=l(R)=R(1-e)$. Also, any total order of $R$ is of the form $(B \oplus C \oplus D)^{+}$. If $C \neq 0$ and $D \neq 0$, then a total order $(C \oplus D)^{+}$of $C \oplus D$ could be extended to a total order of $R$. Thus one of $C$ or $D$ is zero but the other is nonzero. If $C=0$ then $B$ and $D$ are ideals of $R$. If $B$ is not a field and $0 \neq b \in B$ with $b^{2}=0$ and $0 \neq d \in D$, then the partial

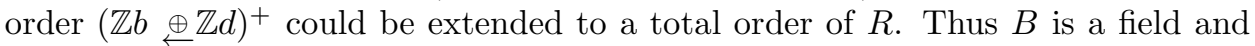
$R$ is of type (i). Suppose then that $D=0$. If $0 \neq b \in B$ with $b^{2}=0$, then, since in any total order of $R$ either $b \geq C$ or $-b \geq C$, we must have $b C=0$. But then for $0 \neq c \in C$ there is a total order of $R$ containing $(\mathbb{Z} b \oplus \mathbb{Z} c)^{+}$. So $B$ is a field. By an argument similar to the one given when $R$ is unital we see that $B=\mathbb{Q}$. Thus $R$ is of type (ii).

We next show that each of these algebras is an $O^{*}$ - algebra. If $R=F \oplus N$ is of type (i) and $P$ is a partial order of $R$, then $P_{F}=\{\alpha \in F: \alpha+x \in P$ for some $x \in N\}$ is a partial order of $F$. For $P_{F}$ is closed under addition and multiplication; and if $\alpha+x$ and $-\alpha+y$ are in $P$ then $-\alpha^{2} \in P \cap F$. Thus $\alpha=0$ since $F$ is an $O^{*}$ - field. Now, if $T_{F}$ is a total order of the field $F$ with $T_{F} \supseteq P_{F}$ and $T_{N}$ is a total order of the group $N$ with $T_{N} \supseteq P \cap N$, then $R^{+}=\left[\left(F, T_{F}\right) \stackrel{\oplus}{\rightarrow}\left(N, T_{N}\right)\right]^{+}$ is a total order of $R$ which contains $P$. 
Suppose that $R$ is of type (iii) and that $P$ is a partial order of $R$. If $x=$ $\left(\begin{array}{ll}a & b \\ 0 & a\end{array}\right) \in P$ with $a<0$ then we may assume that $a=-1$. But then $-1=$ $x^{2}+2 x \in P$ and this is impossible. So if $T_{N}$ is a total order of the group $\left(\begin{array}{ll}0 & N \\ 0 & 0\end{array}\right)$ which contains $\left(\begin{array}{ll}0 & N \\ 0 & 0\end{array}\right) \cap P$, then $\left.\mathbb{Q}\left(\begin{array}{ll}1 & 0 \\ 0 & 1\end{array}\right) \oplus\left(\begin{array}{ll}0 & N \\ 0 & 0\end{array}\right), T_{N}\right)$ gives a total order of $R$ which contains $P$. Similarly, each ring of type (ii) is an $O^{*}$ - ring.

It is interesting to note that the unique totally ordered (right or left) self-injective rings that are not unital are $O^{*}$ - rings [8, Theorem 5.4].

A well-known result of Serre's [4, p.117] implies that a real algebraic extension of $\mathbb{Q}$ is an $O^{*}$ - field if and only if each of the algebraic number fields that it contains is an $O^{*}$ - field.

Example. Each real quadratic extension of $\mathbb{Q}$ is an $O^{*}$ - field.

To see this we may assume that $F=\mathbb{Q}(\sqrt{e})$ where $e \in \mathbb{Z}^{+}$is square-free. Let $P$ be a partial order of $F$. By replacing $P$ by $\mathbb{Q}^{+} P+\mathbb{Q}^{+}$we may assume that $\mathbb{Q}^{+} P \subseteq P$ and $1 \in P$. Now, $F$ has exactly two total orders [6, p. 287]: $T_{1}=F \cap \mathbb{R}^{+}$ and $T_{2}=\left\{a+b \sqrt{e}: a-b \sqrt{e} \in \mathbb{R}^{+}, a, b \in \mathbb{Q}\right\}$ where $\mathbb{R}^{+}$is the total order of $\mathbb{R}$. All of the inequalities that subsequently appear will refer to this total order. If $x=a+b \sqrt{e}$, then $\bar{x}=a-b \sqrt{e}$. We first note that

$(*) \quad$ If $x=a+b \sqrt{e} \in P$ with $a<0$, then $x \bar{x}=a^{2}-b^{2} e<0$ and :

$$
\begin{aligned}
& b>0 \Leftrightarrow \sqrt{e} \in P \Leftrightarrow \bar{x}<0, \\
& b<0 \Leftrightarrow-\sqrt{e} \in P \Leftrightarrow x<0 .
\end{aligned}
$$

For $b \sqrt{e}=x-a \in P$; so $b>0$ (respectively, $b<0) \Leftrightarrow \sqrt{e}$ (respectively, $-\sqrt{e}) \in$ $P$. Also, $x^{2}-a x=e b^{2}+a b \sqrt{e} \in P$; so $1+\frac{a}{e b} \sqrt{e},-\left(1+\frac{b}{a} \sqrt{e}\right) \in P$, and consequently $\left(\frac{a^{2}-b^{2} e}{a b e}\right) \sqrt{e}=\left(\frac{a}{e b}-\frac{b}{a}\right) \sqrt{e} \in P$. Thus, $a^{2}-b^{2} e<0$ in both cases. If $x<0$ and also $b>0$, then $b^{2} e<a^{2}$; so $b<0$. Trivially, $b<0$ gives $x<0$. The other case is similar.

Suppose that $P \nsubseteq T_{1}, T_{2}$. Then there are $x \in P \backslash T_{1}$ and $y \in P \backslash T_{2}$. So $x=a+b \sqrt{e}<0$ and $y=c+d \sqrt{e}$ with $\bar{y}<0$; hence $a<0$ or $b<0$, and $c<0$ or $d>0$. We consider each of the four cases separately.

(I) $a<0$ and $c<0$. This case is impossible by $(* 1)$ and $(* 2)$.

(II) $a<0$ and $d>0$. By $(* 2)-\sqrt{e} \in P$ and hence $c>0$. But then $y_{1}=-\sqrt{e} y=-d e-c \sqrt{e} \in P$ and $\bar{y}_{1}=-d e+c \sqrt{e}=\sqrt{e} \bar{y}<0$. This is case I.

(III) $b<0$ and $c<0$. After passing to $\bar{P}$ this is case II.

(IV) $b<0$ and $d>0$. To avoid the other cases $a \geq 0$ and $c \geq 0$. If $a=0$ then $-\sqrt{e} \in P$, and hence $-\sqrt{e} y=-d e-c \sqrt{e} \in P ;$ so $c^{2} e>d^{2} e^{2}$ and $c^{2}>d^{2} e$ by $(*)$. But $c<d \sqrt{e}$ since $\bar{y}<0$. Thus $a>0$. If $c=0$, then $\sqrt{e} \in P$ and $\sqrt{e} x=b e+a \sqrt{e} \in P$; and hence $y \in P$ gives case II. Thus $c>0$ and $x y=(a c+b d e)+(a d+b d) \sqrt{e} \in P$ with $a c+b d e<0$, since $a<-b \sqrt{e}$ and $c<d \sqrt{e}$. By $(* 1)$ and $(* 2) \sqrt{e} \in P$ or $-\sqrt{e} \in P$. If the former holds then $\sqrt{e} x=b e+a \sqrt{e} \in P$; this is case II. If the latter holds, $y_{1}=-\sqrt{e} y=-d e-c \sqrt{e} \in P$ and $\bar{y}_{1}<0$. This contradicts $(* 1)$. 
This calculation actually gives the

Corollary. The following statements are equivalent for the quadratic extension $F=K(\sqrt{e})$ of the $O^{*}$ - field $K$.

(1) $F$ is an $O^{*}$ - field.

(2) $e$ is totally positive in $K$ (that is, $e$ is positive in each total order of $K$ ), and for each partial order $P$ of $F$ there is a total order $T$ of $K$ such that PT is a partial order of $F$.

(3) Each maximal partial order of $F$ contains e and a total order of $K$.

Note that $\mathbb{Q}(\sqrt[4]{e})$ is not an $O^{*}$ - field if $0<e \in \mathbb{Z}$ is square-free.

\section{REFERENCES}

1. A.A. Albert, On ordered algebras, Bull. Amer. Math. Soc. 46(1940), 521-522. MR 1:328e

2. A. Bigard, K. Keimel, and S. Wolfenstein, Groupes et Anneaux Réticulés, Lecture Notes in Math. 608, Springer-Verlag, New York, 1977. MR 58:27688

3. G. Birkhoff and R.S. Pierce, Lattice-ordered rings, An. Acad. Brasil. Ci. 28(1956), 41-69. MR 18:191d

4. L. Fuchs, Partially ordered algebraic systems, Akademia Kiadó, Budapest, 1963. MR 30:2090

5. M. Henriksen and J.R. Isbell, Lattice-ordered rings and function rings, Pacific J. Math. 12 (1962), 533-565. MR 27:3670

6. N. Jacobson, Lectures in abstract algebra III, Von Nostrand, Princeton, 1964. MR 30:3087

7. V. Kreinovich, If a polynomial identity guarantees that every partial order on a ring can be extended, then this identity is true only for a zero-ring, Algebra Universalis 33 (1995), 237-242. MR 96c:06030

8. S.A. Steinberg, Quotient rings of a class of lattice-ordered rings, Canad. J. Math. 25 (1973), 627-645. MR 49:4901

Department of Mathematics, The University of Toledo, Toledo, Ohio 43606-3390

E-mail address: ssteinb@uoft02.utoledo.edu 